\title{
FAMILIES OF ELLIPTIC CURVES OVER QUARTIC NUMBER FIELDS WITH PRESCRIBED TORSION SUBGROUPS
}

\author{
DAEYEOL JEON, CHANG HEON KIM, AND YOONJIN LEE
}

\begin{abstract}
We construct infinite families of elliptic curves with given torsion group structures over quartic number fields. In a 2006 paper, the first two authors and Park determined all of the group structures which occur infinitely often as the torsion of elliptic curves over quartic number fields. Our result presents explicit examples of their theoretical result. This paper also presents an efficient way of finding such families of elliptic curves with prescribed torsion group structures over quadratic or quartic number fields.
\end{abstract}

\section{INTRODUCTION}

It is an important research problem to determine all of the torsion group structures of elliptic curves $E$ over a number field and to find an infinite family of elliptic curves with a given torsion group structure. We briefly introduce some development on this research problem.

Over the rational number field $\mathbb{Q}$, Mazur 8 ] theoretically characterized all the possible torsion groups of elliptic curves, showing that the torsion group $E(\mathbb{Q})_{\text {tors }}$ of an elliptic curve $E$ over $\mathbb{Q}$ is isomorphic to exactly one of the following 15 types:

$$
\begin{array}{ll}
\mathbb{Z} / N \mathbb{Z}, & N=1-10,12, \\
\mathbb{Z} / 2 \mathbb{Z} \oplus \mathbb{Z} / 2 N^{\prime} \mathbb{Z}, & N^{\prime}=1-4 .
\end{array}
$$

In fact, each of these groups in Eq. (1) appears infinitely often as a torsion group $E(\mathbb{Q})_{\text {tors }}$ of $E$ over $\mathbb{Q}$. In other words, for each of the groups in Eq. (1) there are infinitely many absolutely nonisomorphic elliptic curves with such a torsion group structure over $\mathbb{Q}$. This follows from the fact that the modular curves $X_{1}(N)$ and $X_{1}\left(2,2 N^{\prime}\right)$ parametrizing elliptic curves with such a torsion structure are rational, so they have infinitely many $\mathbb{Q}$-rational points. Kubert [7, Table 3] explicitly parametrized an infinite family of elliptic curves $E$ with such a torsion group structure over $\mathbb{Q}$ for each of the 15 types in Eq. (1).

Received by the editor June 29, 2010 and, in revised form, October 18, 2010.

2010 Mathematics Subject Classification. Primary 11G05; Secondary: 11G18.

Key words and phrases. Elliptic curve, torsion, quadratic number field, quartic number field, modular curve.

The first author was supported by the research grant of the Kongju National University in 2009.

The second author was supported by the Basic Science Research Program through the National Research Foundation of Korea (NRF) funded by the Ministry of Education, Science and Technology (2010-0001654).

The third author is the corresponding author and was supported by Priority Research Centers Program through the National Research Foundation of Korea (NRF) funded by the Ministry of Education, Science and Technology (2009-0093827). 
Over quadratic number fields, Kamienny and Mazur [5] theoretically determined all possible torsion groups of elliptic curves as follows (total 26 types):

$$
\begin{array}{ll}
\mathbb{Z} / N \mathbb{Z}, & N=1-16,18, \\
\mathbb{Z} / 2 \mathbb{Z} \oplus \mathbb{Z} / 2 N^{\prime} \mathbb{Z}, & N^{\prime}=1-6, \\
\mathbb{Z} / 3 \mathbb{Z} \oplus \mathbb{Z} / 3 N^{\prime \prime} \mathbb{Z}, & N^{\prime \prime}=1-2, \\
\mathbb{Z} / 4 \mathbb{Z} \oplus \mathbb{Z} / 4 \mathbb{Z} . &
\end{array}
$$

Again, each of these 26 groups occurs infinitely often as $E(K)_{\text {tors }}$, provided we allow the quadratic number field $K$ to vary as well.

As mentioned previously, over the rational field or quadratic number fields, there was development on characterization of groups which appear infinitely often as torsion groups of elliptic curves. In this vein, it is very natural to investigate which groups would occur infinitely often as torsion groups of elliptic curves over quartic number fields. Recently, the first two authors and Park [3] determined which groups occur infinitely often as torsion groups $E(K)_{\text {tors }}$ when $K$ varies over all quartic number fields and $E$ varies over all elliptic curves over $K$. They proved that all of the group structures occurring infinitely often as torsion groups $E(K)_{\text {tors }}$ are exactly the following 38 types:

$$
\begin{array}{ll}
\mathbb{Z} / N_{1} \mathbb{Z}, & N_{1}=1-18,20,21,22,24, \\
\mathbb{Z} / 2 \mathbb{Z} \oplus \mathbb{Z} / 2 N_{2} \mathbb{Z}, & N_{2}=1-9, \\
\mathbb{Z} / 3 \mathbb{Z} \oplus \mathbb{Z} / 3 N_{3} \mathbb{Z}, & N_{3}=1-3, \\
\mathbb{Z} / 4 \mathbb{Z} \oplus \mathbb{Z} / 4 N_{4} \mathbb{Z}, & N_{4}=1-2, \\
\mathbb{Z} / 5 \mathbb{Z} \oplus \mathbb{Z} / 5 \mathbb{Z}, & \\
\mathbb{Z} / 6 \mathbb{Z} \oplus \mathbb{Z} / 6 \mathbb{Z} . &
\end{array}
$$

The main goal of this paper is to construct explicit examples of the theoretical result in [3, that is to say, the construction of infinite families of elliptic curves with the torsion groups in Eq. (3) over quartic number fields as Kubert did over $\mathbb{Q}$. While the subject of the torsion of elliptic curves over number fields of higher order has been studied by Kamienny and Mazur [5, Merel 9], Parent [12, 13, Zimmer et al. 11, 18, and Jeon et al. 3, 4, there has not been much development for finding elliptic curves with a given torsion group over number fields of higher order. Recently, the cubic number field case is treated in [2, 4]; in 4, all of the group structures which occur infinitely often as the torsion of elliptic curves over cubic number fields are determined, and in 2, we construct infinite families of elliptic curves with given torsion group structures over cubic number fields. It is known [4, Lemma 3.3] that if $E$ is an elliptic curve over $\mathbb{Q}$ and $E^{\prime}$ an elliptic curve over a quadratic number field $k$, then for almost all quadratic number fields $K$ we have $E(K)_{\text {tors }}=E(\mathbb{Q})_{\text {tors }}$, and for almost all quadratic extension $L$ of $k$ we have $E^{\prime}(L)_{\text {tors }}=E^{\prime}(k)_{\text {tors }}$. Due to this fact, the group structure that already occurs over $\mathbb{Q}$ (respectively, quadratic number fields $k$ ) would appear infinitely often over suitable quadratic number fields $K$ (respectively, quartic number fields $L$ ) without increasing the torsion.

In order to achieve our goal, according to the fact mentioned in the previous paragraph, it is sufficient to find infinite families of elliptic curves with prescribed torsion groups which do not occur over $\mathbb{Q}$ (respectively, quadratic number fields) but occur over quadratic number fields (respectively, quartic number fields). Therefore, over quadratic number fields, for each of the following 11 types in Eq. (4), we 
construct an explicit infinite family of elliptic curves with such a torsion group:

$$
\begin{array}{ll}
\mathbb{Z} / N \mathbb{Z}, & N=11,13,14,15,16,18, \\
\mathbb{Z} / 2 \mathbb{Z} \oplus \mathbb{Z} / 2 N^{\prime} \mathbb{Z}, & N^{\prime}=5,6, \\
\mathbb{Z} / 3 \mathbb{Z} \oplus \mathbb{Z} / 3 \mathbb{Z}, & \\
\mathbb{Z} / 3 \mathbb{Z} \oplus \mathbb{Z} / 6 \mathbb{Z}, & \\
\mathbb{Z} / 4 \mathbb{Z} \oplus \mathbb{Z} / 4 \mathbb{Z} . &
\end{array}
$$

On the other hand, over quartic number fields, for each of the following 12 types in Eq. (5), we obtain an explicit infinite family of elliptic curves with such a torsion group:

$$
\begin{array}{ll}
\mathbb{Z} / N \mathbb{Z}, & N=17,20,21,22,24, \\
\mathbb{Z} / 2 \mathbb{Z} \oplus \mathbb{Z} / 2 N^{\prime} \mathbb{Z}, & N^{\prime}=7,8,9, \\
\mathbb{Z} / 3 \mathbb{Z} \oplus \mathbb{Z} / 9 \mathbb{Z}, & \\
\mathbb{Z} / 4 \mathbb{Z} \oplus \mathbb{Z} / 8 \mathbb{Z}, & \\
\mathbb{Z} / 5 \mathbb{Z} \oplus \mathbb{Z} / 5 \mathbb{Z}, & \\
\mathbb{Z} / 6 \mathbb{Z} \oplus \mathbb{Z} / 6 \mathbb{Z} . &
\end{array}
$$

We briefly explain the methods used in this paper. Regarding all of the cyclic torsion group cases, we construct families of elliptic curves with the prescribed cyclic torsion by finding infinitely many quadratic points and quartic points on modular curves $X_{1}(N)$ for $N=11,13-18,20,21,22,24$. For achieving this, we need to search for proper forms of defining equations which yield such quadratic or quartic points. Reichert 14 calculated defining equations of the modular curves $X_{1}(N)$ for $N=11,13-16,18$ by using the Tate normal form. Very recently, Sutherland [17] improved Reichert's result, so he obtained optimized forms (in terms of degree, number of terms, and coefficient size) for defining equations of $X_{1}(N)$ for $N \leq 50$. For all the cyclic torsion cases we consider except for $N=24$, that is, for $N=11,13-18,20-22$, the defining models $X_{1}(N)$ obtained by Sutherland [17, Table 6] are in proper form to use for our purpose. But, his model for $X_{1}(24)$ is not in proper form, so in this case of cyclic torsion $\mathbb{Z} / 24 \mathbb{Z}$, we use instead the forgetful map from $X_{1}(24)$ to $X_{1}(12)$ to construct our family of elliptic curves. On the other hand, for the noncyclic torsion cases, we use Kubert families [7, Table 1 and Table $3]$ and some other methods such as Theorem 2.1 and Proposition 4.8.

This paper is organized as follows. We begin with some basic notions in Section2, Section 3 presents infinite families of elliptic curves over quadratic number fields with torsion groups in Eq. (41), and in Section 4 we find infinite families of elliptic curves over quartic number fields with torsion groups in Eq. (5).

\section{Preliminaries}

In this section we introduce some basic notions on elliptic curves, and we can refer to 1, 6, 7, 16, for more details.

The general normal form of the cubic defining an elliptic curve passing through $P=(0,0)$ is the following:

$$
E: y^{2}+a_{1} x y+a_{3} y=x^{3}+a_{2} x^{2}+a_{4} x .
$$

From the calculation of the derivative $y^{\prime}$ in the relation

$$
\left(2 y+a_{1} x+a_{3}\right) y^{\prime}=3 x^{2}+2 a_{2} x+a_{4}-a_{1} y
$$

we see that the slope of the tangent line at $P$ is $a_{4} / a_{3}$ on $E$, so $E$ is not singular at $P$ if and only if $a_{3} \neq 0$ or $a_{4} \neq 0$. 
Assume that $E$ is nonsingular. Then $P$ is of order 2 if and only if $a_{3}=0$ (and therefore $a_{4} \neq 0$ ), i.e., $E$ has the following equation:

$$
y^{2}+a_{1} x y=x^{3}+a_{2} x^{2}+a_{4} x .
$$

If $a_{3} \neq 0$, then by the admissible change of variables,

$$
x=X, \quad y=Y+a_{3}^{-1} a_{4} X,
$$

the curve $E$ becomes

$$
Y^{2}+\left(a_{1}+2 a_{3}^{-1} a_{4}\right) X Y+a_{3} Y=X^{3}+\left(a_{2}-a_{1} a_{3}^{-1} a_{4}-a_{3}^{-2} a_{4}^{2}\right) X^{2},
$$

which can be rewritten as

$$
E^{\prime}: y^{2}+a_{1} x y+a_{3} y=x^{3}+a_{2} x^{2} .
$$

We have

$$
-P=\left(0,-a_{3}\right), \quad 2 P=\left(-a_{2}, a_{1} a_{2}-a_{3}\right)
$$

by the chord-tangent method [6, Chapter III], thus $3 P=O(O$ denotes the point at infinity) if and only if $-P=2 P$, which implies that $P$ is of order 3 if and only if $a_{2}=0$. Assume that $P$ is not of order 2 or 3 , that is, $a_{2} \neq 0$ and $a_{3} \neq 0$. Under the change of coordinates,

$$
x=X / u^{2}, \quad y=Y / u^{3} \text { with } u=a_{3}^{-1} a_{2},
$$

and letting $b=-a_{3}^{-2} a_{2}^{3}$ and $c=1-a_{3}^{-1} a_{1} a_{2}$, we obtain the Tate normal form of an elliptic curve with $P=(0,0)$ as follows:

$$
E=E(b, c): Y^{2}+(1-c) X Y-b Y=X^{3}-b X^{2},
$$

and this is nonsingular if and only if $b \neq 0$. On the curve $E(b, c)$ we have the following by the chord-tangent method:

$$
\begin{aligned}
P & =(0,0), \\
2 P & =(b, b c), \\
3 P & =(c, b-c), \\
4 P & =\left(r(r-1), r^{2}(c-r+1)\right) ; \quad b=c r, \\
5 P & =\left(r s(s-1), r s^{2}(r-s)\right) ; c=s(r-1), \\
6 P & =\left(\frac{s(r-1)(r-s)}{(s-1)^{2}}, \frac{s^{2}(r-1)^{2}(r s-2 r+1)}{(s-1)^{3}}\right) .
\end{aligned}
$$

Very recently, by using the Tate normal form, Sutherland [17 found optimized forms for defining equations of the modular curves $X_{1}(N)$ for $N=11,13-50$. The formulas in Table 1 and Table 2 are taken directly from [17, Table 6 and Table 7]. We use those defining equations for $N=11,13-18,21,22,24$, which are given in Table 1. We also need Table 2 for birational maps for $X_{1}(N)$ for our purpose.

In fact, the condition $N P=O$ in $E(b, c)$ gives a defining equation for $X_{1}(N)$. For example, $11 P=O$ implies $5 P=-6 P$, so

$$
x_{5 P}=x_{-6 P}=x_{6 P},
$$

where $x_{n P}$ denotes the $x$-coordinate of the $n$-multiple $n P$ of $P$. Eq. (6) implies that

$$
r s(s-1)=\frac{s(r-1)(r-s)}{(s-1)^{2}} .
$$


TABLE 1. Optimized form of $X_{1}(N): f(u, v)=0$

\begin{tabular}{l|l}
$N$ & $f(u, v)$ \\
\hline & \\
11 & $v^{2}+\left(u^{2}+1\right) v+u$ \\
13 & $v^{2}+\left(u^{3}+u^{2}+1\right) v-u^{2}-u$ \\
14 & $v^{2}+\left(u^{2}+u\right) v+u$ \\
15 & $v^{2}+\left(u^{2}+u+1\right) v+u^{2}$ \\
16 & $v^{2}+\left(u^{3}+u^{2}-u+1\right) v+u^{2}$ \\
17 & $v^{4}+\left(u^{3}+u^{2}-u+2\right) v^{3}+\left(u^{3}-3 u+1\right) v^{2}-\left(u^{4}+2 u\right) v+u^{3}+u^{2}$ \\
18 & $v^{2}+\left(u^{3}-2 u^{2}+3 u+1\right) v+2 u$ \\
20 & $v^{3}+\left(u^{2}+3\right) v^{2}+\left(u^{3}+4\right) v+2$ \\
21 & $v^{4}+\left(3 u^{2}+1\right) v^{3}+\left(u^{5}+u^{4}+2 u^{2}+2 u\right) v^{2}+\left(2 u^{4}+u^{3}+u\right) v+u^{3}$ \\
22 & $v^{4}+\left(u^{3}+2 u^{2}+u+2\right) v^{3}+\left(u^{5}+u^{4}+2 u^{3}+2 u^{2}+1\right) v^{2}$ \\
& $+\left(u^{5}-u^{4}-2 u^{3}-u^{2}-u\right) v-u^{4}-u^{3}$ \\
24 & $v^{5}+\left(u^{4}+4 u^{3}+3 u^{2}-u-2\right) v^{4}-\left(2 u^{4}+8 u^{3}+7 u^{2}-1\right) v^{3}$ \\
& $-\left(2 u^{5}+4 u^{4}-3 u^{3}-5 u^{2}-u\right) v^{2}+\left(2 u^{5}+5 u^{4}+2 u^{3}\right) v+u^{6}+u^{5}$
\end{tabular}

TABle 2. Birational maps $\varphi$ for $X_{1}(N)$ from $f(u, v)=0$ to $F(r, s)=0$

\begin{tabular}{l|ll}
$N$ & $\varphi$ & \\
\hline 11 & $r=1+u v$, & $s=1-u$ \\
13 & $r=1-u v$, & $s=1-\frac{u v}{v+1}$ \\
14 & $r=1-\frac{u+v}{(v+1)(u+v+1)}$, & $s=\frac{1-u}{v+1}$ \\
15 & $r=1+\frac{u v+v^{2}}{\left(u^{3}+u^{2} v+u^{2}\right)}$, & $s=1+\frac{v}{u^{2}+u}$ \\
16 & $r=\frac{u^{2}-u v+v^{2}+v}{u^{2}+u-v-1}$, & $s=\frac{u-v}{u+1}$ \\
17 & $r=\frac{u^{2}+u-v}{u^{2}+u v+u-v^{2}-v}$, & $s=\frac{u+1}{u+v+1}$ \\
18 & $r=\frac{u^{2}-u v-3 u+1}{(u-1)^{2}(u v+1)}$, & $s=\frac{u^{2}-2 u-v}{u^{2}-u v-3 u-v^{2}-2 v}$ \\
20 & $r=1+\frac{u^{3}+u v+u}{(u-1)^{2}\left(u^{2}-u+v+1\right)}$, & $s=1+\frac{u^{2}+v+1}{(u-1)\left(u^{2}-u+v+2\right)}$ \\
21 & $r=1+\frac{\left(v^{2}+v\right)(u v+v+1)}{(u v+1)\left(u v-v^{2}+1\right)}$, & $s=1+\frac{v^{2}+v}{u v+1}$ \\
22 & $r=\frac{u^{2} v+u^{2}+u v+v}{u^{3}+2 u^{2}+v}$, & $s=\frac{u v+v}{u^{2}+v}$ \\
24 & $r=\frac{u^{2}+u-v+1}{u^{2}+u v-v^{2}+v}$, & $s=\frac{u+1}{u+v}$
\end{tabular}

Without loss of generality, the cases $s=1$ and $s=0$ may be excluded. Then Eq. (17) becomes

$$
r^{2}-4 s r+3 s^{2} r-s^{3} r+s=0
$$

which is one of the equations $X_{1}(11)$, called the raw form of $X_{1}(11)$. By the coordinate changes $s=1-u$ and $r=1+u v$, we get the following equation:

$$
v^{2}+\left(u^{2}+1\right) v+u=0 .
$$

The following well-known theorem [6, Theorem 4.2] provides us with the condition for the divisibility of a given point on $E$ by 2 , and this result is very useful for studying torsion subgroups of the form $\mathbb{Z} / 2 \mathbb{Z} \oplus \mathbb{Z} / 2 N \mathbb{Z}$. 
TABle 3. Polynomials $d_{N}=d_{N}(t)$ and coefficients $b_{N}, c_{N}$

\begin{tabular}{|c|c|}
\hline$N$ & Polynomials $d_{N}=d_{N}(t)$ and coefficients $b_{N}, c_{N}$ \\
\hline 11 & $\left\{\begin{array}{l}b_{11}=t^{4}+2 t^{2}-4 t+1 \\
b_{11}=\frac{t(t-1)\left(t^{2}+1-\sqrt{d_{11}}\right)\left(t^{3}+t-2-t \sqrt{d_{11}}\right)}{4} \\
c_{1}\end{array}\right.$ \\
\hline 13 & $\left\{\begin{array}{l}d_{13}=t^{6}+2 t^{5}+t^{4}+2 t^{3}+6 t^{2}+4 t+1 \\
b_{13}=-\frac{t\left(t^{4}-t^{2}+t+1-(t-1) \sqrt{d_{13}}\right)\left(t^{3}+t^{2}+1-\sqrt{d_{13}}\right)\left(t^{4}+t^{3}+t+2-t \sqrt{d_{13}}\right)}{4\left(t^{3}+t^{2}-1-\sqrt{d_{13}}\right)} \\
c_{13}=-\frac{t\left(t^{3}+t^{2}+1-\sqrt{d_{13}}\right)\left(t^{4}-t^{2}+t+1-(t-1) \sqrt{d_{13}}\right)}{2\left(t^{3}+t^{2}-1-\sqrt{d_{13}}\right)}\end{array}\right.$ \\
\hline 14 & $\begin{array}{l}d_{14}=t^{4}+2 t^{3}+t^{2}-4 t \\
\left\{\begin{array}{l}b_{14}=\frac{8(t-1)\left(t^{2}-t-\sqrt{d_{14}}\right)\left(t^{4}+t^{3}-t^{2}-3 t+2-\left(t^{2}-1\right) \sqrt{d_{14}}\right)}{\left(t^{2}+t-2-\sqrt{d_{14}}\right)^{3}\left(t^{2}-t-2-\sqrt{d_{14}}\right)^{2}} \\
c_{14}=\frac{4(t-1)\left(t^{2}-t-\sqrt{d_{14}}\right)}{\left(t^{2}+t-2-\sqrt{d_{14}}\right)^{2}\left(t^{2}-t-2-\sqrt{d_{14}}\right)}\end{array}\right.\end{array}$ \\
\hline 15 & $\left\{\begin{array}{l}d_{15}=t^{4}+2 t^{3}-t^{2}+2 t+1 \\
b_{15}=\frac{\left(t^{2}+t-1+\sqrt{d_{15}}\right)\left(t^{2}+t+1-\sqrt{d_{15}}\right)\left(t^{2}-t+1-\sqrt{d_{15}}\right)\left(2 t^{3}+t^{2}+t+1-\sqrt{d_{15}}\right)}{4 t^{5}(t+1)\left(t^{2}-t-1-\sqrt{d_{15}}\right)^{2}} \\
c_{15}=-\frac{\left(t^{2}-t+1-\sqrt{d_{15}}\right)\left(t^{2}+t+1-\sqrt{d_{15}}\right)\left(t^{2}+t-1+\sqrt{d_{15}}\right)}{4 t^{3}(t+1)\left(t^{2}-t-1-\sqrt{d_{15}}\right)}\end{array}\right.$ \\
\hline 16 & $\left\{\begin{array}{l}d_{16}=t^{6}+2 t^{5}-t^{4}-t^{2}-2 t+1 \\
b_{16}=\frac{t^{2}\left(t^{3}+t^{2}-t+1-\sqrt{d_{16}}\right)\left(t^{6}+2 t^{5}-t^{3}-2 t^{2}-t+1-\left(t^{3}+t^{2}-1\right) \sqrt{d_{16}}\right)\left(t^{3}+t^{2}+t+1-\sqrt{d_{16}}\right)}{2\left(t^{3}+3 t^{2}+t-1-\sqrt{d_{16}}\right)^{2}} \\
c_{16}=\frac{\left(t^{6}+2 t^{5}-t^{3}-2 t^{2}-t+1-\left(t^{3}+t^{2}-1\right) \sqrt{d_{16}}\right)\left(t^{3}+t^{2}+t+1-\sqrt{d_{16}}\right)}{2(t+1)\left(t^{3}+3 t^{2}+t-1-\sqrt{d_{16}}\right)}\end{array}\right.$ \\
\hline 18 & $\begin{array}{l}d_{18}=t^{6}-4 t^{5}+10 t^{4}-10 t^{3}+5 t^{2}-2 t+1 \\
\left\{\begin{array}{l}b_{18}=-\frac{t\left(t^{3}-t+1-\sqrt{d_{18}}\right)\left(t^{5}-4 t^{4}+9 t^{3}-9 t^{2}+4 t-\left(t^{2}-2 t+2\right) \sqrt{d_{18}}\right)\left(t^{4}-2 t^{3}+5 t^{2}-5 t+2-t \sqrt{d_{18}}\right)}{(t-1)^{4}\left(t^{6}-4 t^{5}+9 t^{4}-10 t^{3}+4 t^{2}+t-1-\left(t^{3}-2 t^{2}+2 t-1\right) \sqrt{d_{18}}\right)\left(t^{4}-2 t^{3}+3 t^{2}+t-2-t \sqrt{d_{18}}\right)^{2}} \\
c_{18}=\frac{t\left(t^{5}-4 t^{4}+9 t^{3}-9 t^{2}+4 t-\left(t^{2}-2 t+2\right) \sqrt{d_{18}}\right)\left(t^{3}-t+1-\sqrt{d_{18}}\right)}{(t-1)^{2}\left(t^{6}-4 t^{5}+9 t^{4}-10 t^{3}+4 t^{2}+t-1-\left(t^{3}-2 t^{2}+2 t-1\right) \sqrt{d_{18}}\right)\left(t^{4}-2 t^{3}+3 t^{2}+t-2-t \sqrt{d_{18}}\right)}\end{array}\right.\end{array}$ \\
\hline
\end{tabular}

Theorem 2.1. Let $E$ be an elliptic curve defined over a field $k$ of characteristic $\neq 2,3$ given by

$$
y^{2}=(x-\alpha)(x-\beta)(x-\gamma)
$$

with $\alpha, \beta, \gamma$ in $k$. For $\left(x_{2}, y_{2}\right)$ in $E(k)$ there exists $\left(x_{1}, y_{1}\right)$ in $E(k)$ such that $2\left(x_{1}, y_{1}\right)=\left(x_{2}, y_{2}\right)$ if and only if $x_{2}-\alpha, x_{2}-\beta$ and $x_{2}-\gamma$ are squares in $k$.

\section{TORSION SUBGROUPS OVER QUADRATIC NUMBER FIELDS}

Throughout this section, let $K$ be a quadratic number field. Our goal in this section is to construct some families of elliptic curves with prescribed torsion over quadratic number fields which do not occur over $\mathbb{Q}$. Note that by finding the quadratic points of $X_{1}(N)$ we can find the elliptic curve with $N$-torsion point over quadratic number fields. For the cyclic torsion group cases, we obtain families of elliptic curves by calculating the quadratic points satisfying the equations of $X_{1}(N)$ in Table 1.

3.1. The case $E(K)_{\text {tors }}=\mathbb{Z} / N \mathbb{Z}$ with $N=11,13,14,15,16,18$.

Theorem 3.1. For each $N=11,13,14,15,16,18$, let $b_{N}, c_{N}, d_{N}$ be defined as in Table 3. Choose $t \in \mathbb{Q}$ such that the discriminant of the following cubic equation is nonzero:

$$
y^{2}+\left(1-c_{N}\right) x y-b_{N} y=x^{3}-b_{N} x^{2} .
$$

Let $E$ be an elliptic curve defined by the above equation. Then the torsion subgroup of $E$ over a quadratic number field $\mathbb{Q}\left(\sqrt{d_{N}}\right)$ is equal to $\mathbb{Z} / N \mathbb{Z}$.

Proof. We first prove this theorem for the case $N=11$. Note that

$$
(u, v)=\left(t, \frac{-t^{2}-1+\sqrt{t^{4}+2 t^{2}-4 t+1}}{2}\right)
$$


satisfies the defining equation $v^{2}+\left(u^{2}+1\right) v+u=0$ of $X_{1}(11)$ in Table 1. From the birational map in Table 2, we know that $b_{11}$ and $c_{11}$ are expressed as

$$
b_{11}=u(u-1) v(u v+1), c_{11}=u(u-1) v .
$$

The result follows from the substitution $u=t$ and $v=\frac{-t^{2}-1+\sqrt{t^{4}+2 t^{2}-4 t+1}}{2}$. But, in fact, this curve $E$ over $\mathbb{Q}\left(\sqrt{d_{11}}\right)$ has no other torsion points since Kamienny and Mazur [5] determined all possible torsion structures over quadratic number fields.

The other cases can be proved by using the formulas of Table 1 and Table 2 and applying the same method as the case $N=11$.

3.2. The case $E(K)_{\text {tors }}=\mathbb{Z} / 2 \mathbb{Z} \oplus \mathbb{Z} / 10 \mathbb{Z}$.

Theorem 3.2. Put $d=d(t)=8 t^{3}-8 t^{2}+1$ with $t \in \mathbb{Q}$. Let $E$ be an elliptic curve defined by the equation

$$
E: y^{2}+(1-c) x y-b y=x^{3}-b x^{2},
$$

where $b=\frac{t^{3}\left(2 t^{2}-3 t+1\right)}{\left(t^{2}-3 t+1\right)^{2}}$ and $c=-\frac{t\left(2 t^{2}-3 t+1\right)}{t^{2}-3 t+1}$ with $t \neq 0, \frac{1}{2}$, 1 . Then the torsion subgroup of $E$ over $\mathbb{Q}(\sqrt{d})$ is equal to $\mathbb{Z} / 2 \mathbb{Z} \oplus \mathbb{Z} / 10 \mathbb{Z}$.

Proof. The elliptic curve $E$ defined by the form in Eq. (8) has a $\mathbb{Q}$-rational point $P=(0,0)$ of order 10 [7. If we write Eq. (8) as $y^{2}=f(x)$, where $f(x)$ is a cubic polynomial, we see that $f(x)$ has a linear factor over $\mathbb{Q}$ because $E$ has a $\mathbb{Q}$-rational point $5 P$ of order 2 . Then $E$ has full 2-torsion over a number field $L$ if and only if $f(x)$ splits over $L$, equivalently the discriminant of $f(x)$ being a square in $L$. Since the discriminant $\Delta$ of $E$ is 16 times the discriminant of $f(x), E$ has full 2-torsion over $L$ if and only if $\Delta$ is a square in $L$. In fact, $\Delta$ is a square in $L$ if and only if $d=8 t^{3}-8 t^{2}+1$ is a square in $L$, so this implies that the torsion subgroup of $E$ is equal to $\mathbb{Z} / 2 \mathbb{Z} \times \mathbb{Z} / 10 \mathbb{Z}$ over $\mathbb{Q}(\sqrt{d})$.

3.3. The case $E(K)_{\text {tors }}=\mathbb{Z} / 2 \mathbb{Z} \oplus \mathbb{Z} / 12 \mathbb{Z}$.

Theorem 3.3. Put $d=d(t)=\frac{t^{2}-1}{t^{2}+3}$ with $t \in \mathbb{Q}$. Let $E$ be an elliptic curve defined by the equation

$$
y^{2}+(1-c) x y-\left(c+c^{2}\right) y=x^{3}-\left(c+c^{2}\right) x^{2},
$$

where $c=\frac{1-t^{2}}{t^{4}+3 t^{2}}$ with $t \neq-1,0,1$. Then the torsion subgroup of $E$ over $\mathbb{Q}(\sqrt{d})$ is equal to $\mathbb{Z} / 2 \mathbb{Z} \oplus \mathbb{Z} / 12 \mathbb{Z}$.

Proof. The Tate normal form of an elliptic curve $E$ with $\mathbb{Q}$-rational point $(0,0)$ of order 6 is the elliptic curve defined by

$$
y^{2}+(1-c) x y-\left(c+c^{2}\right) y=x^{3}-\left(c+c^{2}\right) x^{2} .
$$

By the coordinate changes $x \rightarrow x$ and $y \rightarrow y+\frac{(c-1)}{2} x+\frac{\left(c^{2}+c\right)}{2}$, we get the following form:

$$
y^{2}=x^{3}-\frac{3 c^{2}+3 c-1}{4} x^{2}+\frac{c^{3}-c}{2} x+\frac{c^{4}+2 c^{3}+c^{2}}{4} .
$$

By substituting $c=\frac{10-2 \alpha}{\alpha^{2}-9}$, we have

$$
y^{2}=\left(x+\frac{2(\alpha-1)^{2}}{(\alpha+3)^{2}(\alpha-3)}\right)\left(x+\frac{2(\alpha-5)}{(\alpha-3)(\alpha+3)}\right)\left(x+\frac{(\alpha-5)(\alpha-1)^{2}}{4(\alpha+3)(\alpha-3)^{2}}\right) .
$$


Note that the point $P=\left(0, \frac{c^{2}+c}{2}\right)$ is of order 6 of the elliptic curve defined by the above equation. By Theorem 2.1 , for a number field $L$, there exists an $L$-rational point $Q$ with $2 Q=P$ if and only if $\frac{2}{\alpha-3}$ and $\frac{\alpha-5}{\alpha+3}$ are square in $L$. Put $\alpha=2 t^{2}+3$. Then $c=\frac{1-t^{2}}{t^{4}+3 t^{2}}$, and $\frac{2}{\alpha-3}=\frac{1}{t^{2}}$ and $\frac{\alpha-5}{\alpha+3}=\frac{t^{2}-1}{t^{2}+3}$ are squares in $\mathbb{Q}(\sqrt{d})$.

3.4. The case $E(K)_{\text {tors }}=\mathbb{Z} / 3 \mathbb{Z} \oplus \mathbb{Z} / 3 \mathbb{Z}$. A one-parameter family of elliptic curves with points of order 3, called the Hessian Family, is given as follows 1, Ch. 4, Section 2]:

$$
X^{3}+Y^{3}+Z^{3}=3 \mu X Y Z,
$$

with $\mu$ in $\mathbb{Q}$. From [7, Table 1], we obtain the following:

Theorem 3.4. Let $K=\mathbb{Q}(\sqrt{-3})=\mathbb{Q}\left(\zeta_{3}\right)$ with a primitive cube root of unity $\zeta_{3}$. Let $E$ be an elliptic curve defined by the following equation:

$$
X^{3}+Y^{3}+Z^{3}=3 t X Y Z,
$$

where $t \in \mathbb{Q}$ with $t^{3} \neq 1$. Then the torsion subgroup of $E$ over $K$ contains $\mathbb{Z} / 3 \mathbb{Z} \oplus$ $\mathbb{Z} / 3 \mathbb{Z}$.

3.5. The case $E(K)_{\text {tors }}=\mathbb{Z} / 3 \mathbb{Z} \oplus \mathbb{Z} / 6 \mathbb{Z}$. For finding a family of elliptic curves with $\mathbb{Z} / 3 \mathbb{Z} \oplus \mathbb{Z} / 6 \mathbb{Z}$ as their torsion group, we begin with the curves in Eq. (9).

Theorem 3.5. Let $K=\mathbb{Q}(\sqrt{-3})=\mathbb{Q}\left(\zeta_{3}\right)$ with a primitive cube root of unity $\zeta_{3}$. Let $E$ be an elliptic curve defined by the equation

$$
X^{3}+Y^{3}+Z^{3}=3 \mu X Y Z,
$$

where $\mu=\frac{2 t^{3}+1}{3 t^{2}}$ and $t \in \mathbb{Q}$ with $t \neq 0,1,-\frac{1}{2}$. Then the torsion subgroup of $E$ over $K$ is equal to $\mathbb{Z} / 3 \mathbb{Z} \oplus \mathbb{Z} / 6 \mathbb{Z}$.

Proof. We can refer to [7, Table 1].

3.6. The case $E(K)_{\text {tors }}=\mathbb{Z} / 4 \mathbb{Z} \oplus \mathbb{Z} / 4 \mathbb{Z}$.

Theorem 3.6. Let $K=\mathbb{Q}(\sqrt{-1})$ and let $E$ be an elliptic curve defined by the equation

$$
y^{2}+x y-\left(\nu^{2}-\frac{1}{16}\right) y=x^{3}-\left(\nu^{2}-\frac{1}{16}\right) x^{2},
$$

where $\nu=t^{2}$ and $t \in \mathbb{Q}$ with $t \neq 0, \pm \frac{1}{16}$. Then the torsion subgroup of $E$ is equal to $\mathbb{Z} / 4 \mathbb{Z} \oplus \mathbb{Z} / 4 \mathbb{Z}$ over $K$.

Proof. We know that the curve $E: y^{2}+x y-\left(\nu^{2}-\frac{1}{16}\right) y=x^{3}-\left(\nu^{2}-\frac{1}{16}\right) x^{2}$ with a parameter $\nu$ has $\mathbb{Z} / 4 \mathbb{Z} \oplus \mathbb{Z} / 2 \mathbb{Z}$ as the torsion group over $\mathbb{Q}$ from [7, Table 1]. We note that $P=(0,0)$ is a point of order 4 and $2 P=\left(\nu^{2}-\frac{1}{16}, 0\right)$. By Theorem 2.1 for another 2-torsion point $Q=\left(-\frac{1}{8}+\frac{\nu}{2}, \frac{(4 \nu-1)^{2}}{32}\right)$, there exist a $K$-rational point $R$ with $2 R=Q$ if and only if $\left(-\frac{1}{8}+\frac{\nu}{2}\right)-\left(-\frac{1}{8}-\frac{\nu}{2}\right)=\nu$ and $\left(-\frac{1}{8}+\frac{\nu}{2}\right)-\left(\nu^{2}-\frac{1}{16}\right)=-\frac{(4 \nu-1)^{2}}{16}$ are squares in $K$. It follows from taking $\nu=t^{2}$. 
TABLE 4. Polynomials $f_{N}(x, t)$ and coefficients $b_{N}, c_{N}$

\begin{tabular}{|c|c|}
\hline$N$ & Polynomials $f_{N}(x, t)$ and coefficients $b_{N}, c_{N}$ \\
\hline 17 & $\begin{array}{l}f_{17}(x, t)=x^{4}+\left(t^{3}+t^{2}-t+2\right) x^{3}+\left(t^{3}-3 t+1\right) x^{2}-\left(t^{4}+2 t\right) x+t^{3}+t^{2} \\
\left\{\begin{array}{l}b_{17}=-\frac{(t+1) \alpha_{t}\left(\alpha_{t}-t\right)\left(\alpha_{t}-t^{2}-t\right)}{\left(\alpha_{t}+t+1\right)\left(\alpha_{t}{ }^{2}-(t-1) \alpha_{t}-t^{2}-t\right)^{2}} \\
c_{17}=-\frac{(t+1) \alpha_{t}\left(\alpha_{t}-t\right)}{\left(\alpha_{t}+t+1\right)\left(\alpha_{t}{ }^{2}-(t-1) \alpha_{t}-t^{2}-t\right)}\end{array}\right.\end{array}$ \\
\hline 20 & 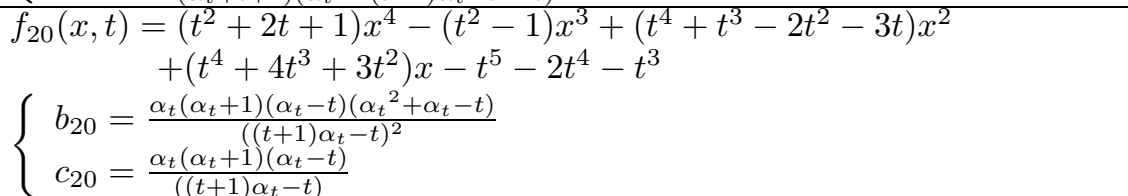 \\
\hline 21 & $\begin{array}{l}f_{21}(x, t)=x^{4}+\left(3 t^{2}+1\right) x^{3}+\left(t^{5}+t^{4}+2 t^{2}+2 t\right) x^{2}+\left(2 t^{4}+t^{3}+t\right) x+t^{3} \\
\left\{\begin{array}{l}b_{21}=\frac{\alpha_{t}\left(\alpha_{t}+1\right)\left((t+1) \alpha_{t}+1\right)\left(\alpha_{t}{ }^{2}+(t+1) \alpha_{t}+1\right)\left(\alpha_{t}{ }^{3}+\left(t^{2}+t+1\right) \alpha_{t}{ }^{2}+(2 t+1) \alpha_{t}+1\right)}{\left(t \alpha_{t}+1\right)^{3}\left(\alpha_{t}{ }^{2}-t \alpha_{t}-1\right)^{2}} \\
c_{21}=-\frac{\alpha_{t}\left(\alpha_{t}+1\right)\left((t+1) \alpha_{t}+1\right)\left(\alpha_{t}{ }^{2}+(t+1) \alpha_{t}+1\right)}{\left(t \alpha_{t}+1\right)^{2}\left(\alpha_{t}{ }^{2}-t \alpha_{t}-1\right)}\end{array}\right.\end{array}$ \\
\hline 22 & $\begin{array}{l}f_{22}(x, t)=x^{4}+\left(t^{3}+2 t^{2}+t+2\right) x^{3}+\left(t^{5}+t^{4}+2 t^{3}+2 t^{2}+1\right) x^{2} \\
\quad+\left(t^{5}-t^{4}-2 t^{3}-t^{2}-t\right) x-t^{4}-t^{3} \\
\left\{\begin{aligned} b_{22}= & \frac{t(t+1) \alpha_{t}\left((t+1) \alpha_{t}-t^{2}-t\right)\left(\left(t^{2}+t+1\right) \alpha_{t}+t^{2}\right)}{\left(\alpha_{t}+t^{2}\right)\left(\alpha_{t}+t^{3}+2 t^{2}\right)^{2}} \\
c_{22}= & \frac{t(t+1) \alpha_{t}\left((t+1) \alpha_{t}-t^{2}-t\right)}{\left(\alpha_{t}+t^{2}\right)\left(\alpha_{t}+t^{3}+2 t^{2}\right)}\end{aligned}\right.\end{array}$ \\
\hline
\end{tabular}

\section{Torsion SUbGroups OVER QUARTIC NUMBER FIELDS $K$}

Throughout this section, $K$ denotes a quartic number field. In this section we construct some families of elliptic curves with prescribed torsion structures given in Eq. (5) over quartic number fields; those torsion structures do not occur over $\mathbb{Q}$ and quadratic number fields.

A smooth projective curve $X$ over an algebraically closed field is called $d$-gonal if there exists a finite morphism $f: X \rightarrow \mathbb{P}^{1}$ of degree $d$. For $d=4$ we say that the curve is tetragonal. Also, the smallest possible $d$ is called the gonality of the curve $X$ and we denote it by $\operatorname{Gon}(X)$. Sutherland [17] basically attempted to find a plane model $f_{N}(x, y)=0$ of $X_{1}(N)$ which minimizes the degree $d$ of one of its variables. Noting that $\operatorname{Gon}\left(X_{1}(20)\right)=3$ (refer to [4]) and $\operatorname{Gon}\left(X_{1}(N)\right)=4$ with $N=17,21,22,24$ (refer to [3]), for the cases $N=17,20,21,22$, Sutherland succeeded in finding plane models $f_{N}(x, y)=0$ such that the degree in $y$ of $f_{N}(x, y)$ is equal to $\operatorname{Gon}\left(X_{1}(N)\right)$. However, the case $N=24$ has not been achieved by Sutherland.

In this section, for $N=17,21,22$, using Sutherland's plane models for $X_{1}(N)$, we find infinite families of elliptic curves over quartic number fields whose torsion is $\mathbb{Z} / N \mathbb{Z}$. For the case $N=20$, we point out that we cannot use Sutherland's plane model for $X_{1}(20)$ since each degree of its variables in his model is not 4 , but 3 . We resolve this case by finding a proper model of $X_{1}(20)$ for our purpose. But, as mentioned before, for the case $N=24$, we need to develop another method for this case, and this case is resolved in the subsection 4.2 .

4.1. The case $E(K)_{\text {tors }}=\mathbb{Z} / N \mathbb{Z}$ with $N=17,20,21,22$. Applying the same method used for the proof of Theorem 3.1 with the formulas of Table 1 and Table 2 . we obtain the following result: 
Theorem 4.1. For each $N=17,20,21,22$, choose $t \in \mathbb{Q}$ such that the corresponding polynomial $f_{N}(x, t)$ in Table 4 is irreducible over $\mathbb{Q}$. Let $\alpha_{t}$ be a zero of $f_{N}(x, t)$. Let $E$ be an elliptic curve defined by the following equation:

$$
y^{2}+\left(1-c_{N}\right) x y-b_{N} y=x^{3}-b_{N} x^{2} .
$$

Then the torsion subgroup of $E$ over a quartic number field $\mathbb{Q}\left(\alpha_{t}\right)$ is equal to $\mathbb{Z} / N \mathbb{Z}$ for almost all $t$.

Remark 4.2. (1) In the above theorem, there are indeed infinitely many values $t \in \mathbb{Q}$ such that the polynomial $f_{N}(x, t)$ in Table 4 is irreducible over $\mathbb{Q}$ by Hilbert's irreducibility theorem.

(2) For each $N=17,20,21,22$, we obtain the elliptic curves E over $\mathbb{Q}\left(\alpha_{t}\right)$ whose torsion groups contain $\mathbb{Z} / N \mathbb{Z}$. But, in fact, these curves $\mathrm{E}$ over $\mathbb{Q}\left(\alpha_{t}\right)$ have no other torsion points for almost all $t$ since Jeon et al. 3 determined all possible torsion structures that occur infinitely often over quartic number fields.

(3) As for a plane model of $X_{1}(20)$ where the degree of one of its variables is equal to 4 , we use the equation

$\left(u^{2}+2 u+1\right) v^{4}-\left(u^{2}-1\right) v^{3}+\left(u^{4}+u^{3}-2 u^{2}-3 u\right) v^{2}+\left(u^{4}+4 u^{3}+3 u^{2}\right) v-u^{5}-2 u^{4}-u^{3}=0$, which is obtained by using the Reichert's method [14].

4.2. The case $E(K)_{\text {tors }}=\mathbb{Z} / 24 \mathbb{Z}$. In this subsection, we construct an infinite family of elliptic curves over quartic number fields whose torsion group is $\mathbb{Z} / 24 \mathbb{Z}$. Since there is a forgetful map of degree 4 from $X_{1}(24)$ to $X_{1}(12)$ which is rational, the points on $X_{1}(24)$ lying above each $\mathbb{Q}$-rational point on $X_{1}(12)$ are automatically defined over quartic number fields. It means that the elliptic curve corresponding to each $\mathbb{Q}$-rational point on $X_{1}(12)$ should have a 24 -torsion point over a quartic number field. We explain the computation process explicitly in the proof of Theorem 4.3 .

Theorem 4.3. Let $f_{24}(x, t)=c_{4}(t) x^{4}+c_{2}(t) x^{2}+c_{1}(t) x+c_{0}(t)$, where

$$
\begin{aligned}
c_{4}(t)= & 16 t^{12}-192 t^{11}+1056 t^{10}-3520 t^{9}+7920 t^{8}-12672 t^{7}+14784 t^{6}-12672 t^{5}+7920 t^{4}-3520 t^{3} \\
& +1056 t^{2}-192 t+16 \\
c_{2}(t)= & 96 t^{14}-1536 t^{13}+9888 t^{12}-36192 t^{11}+86400 t^{10}-144096 t^{9}+174048 t^{8}-154656 t^{7}+100984 t^{6} \\
& \quad-47472 t^{5}+15240 t^{4}-2912 t^{3}+168 t^{2}+48 t-8, \\
c_{1}(t)=- & 768 t^{14}+8064 t^{13}-39040 t^{12}+115520 t^{11}-233408 t^{10}+340544 t^{9}-369664 t^{8}+302720 t^{7} \\
\quad & \quad 187264 t^{6}+86528 t^{5}-29056 t^{4}+6720 t^{3}-960 t^{2}+64 t
\end{aligned}
$$

Choose $t \in \mathbb{Q}$ such that $f_{24}(x, t)$ is irreducible over $\mathbb{Q}$, and let $\alpha_{t}$ be a zero of $f_{24}(x, t)$. Let $E$ be an elliptic curve defined by the equation

$$
y^{2}+(1-c) x y-b y=x^{3}-b x^{2},
$$

where

$$
\left\{\begin{array}{l}
b=\frac{t(2 t-1)\left(3 t^{2}-3 t+1\right)\left(2 t^{2}-2 t+1\right)}{(t-1)^{4}}, \\
c=-\frac{t(2 t-1)\left(3 t^{2}-3 t+1\right)}{(t-1)^{3}} .
\end{array}\right.
$$

Then the torsion subgroup of $E$ over a quartic number field $K=\mathbb{Q}\left(\alpha_{t}\right)$ is equal to $\mathbb{Z} / 24 \mathbb{Z}$ for almost all $t$. 
Proof. The elliptic curve defined as above has a $\mathbb{Q}$-rational torsion point $P=(0,0)$ of order 12. By the coordinate changes $x \rightarrow x$ and $y \rightarrow y+\frac{c-1}{2} x+\frac{b}{2}, E$ is changed to the following form:

$$
y^{2}=x^{3}+\frac{(c-1)^{2}-4 b}{4} x^{2}+\frac{b(c-1)}{2} x+\frac{b^{2}}{4} .
$$

For simplicity, we write the curve in Eq. (10) by

$$
y^{2}=x^{3}+A x^{2}+B x+C,
$$

where $A=\frac{(c-1)^{2}-4 b}{4}, B=\frac{b(c-1)}{2}$, and $C=\frac{b^{2}}{4}$.

Let $P=\left(x_{0}, y_{0}\right)$ be a rational 12-torsion point of the curve in Eq. (11). Changing variables in $x$, we may assume $x_{0}=0$. Then

$$
y_{0}^{2}=C .
$$

Now consider a point $\left(x_{1}, y_{1}\right)$ with $2\left(x_{1}, y_{1}\right)=\left(0, y_{0}\right)$. Take $y=k x+y_{0}$ as the line through $\left(0, y_{0}\right)$ tangent at the unknown point $\left(x_{1}, y_{1}\right)$. Then the three roots of

$$
x^{3}+A x^{2}+B x+C-\left(k x+y_{0}\right)^{2}
$$

are $0, x_{1}$ and $x_{1}$, i.e., $x_{1}$ is a double root of Eq. (12). Thus

$$
\frac{x^{3}+A x^{2}+B x+C-\left(k x+y_{0}\right)^{2}}{x}=\left(x-x_{1}\right)^{2},
$$

and hence the discriminant of

$$
x^{2}+\left(A-k^{2}\right) x+\left(B-2 k y_{0}\right)
$$

is equal to 0 , i.e.,

$$
\left(A-k^{2}\right)^{2}-4\left(B-2 k y_{0}\right)=0,
$$

which is a quartic equation in $k$.

Let $k_{0}$ be a root of Eq. (14) and $K$ a quartic number field containing $k_{0}$. Then

$$
x_{1}=\frac{k_{0}^{2}-A}{2}
$$

is a double root of Eq. (13) and hence also of Eq. (12). Consequently, $2\left(x_{1}, k_{0} x_{1}+\right.$ $\left.y_{0}\right)=\left(0,-y_{0}\right)$, and $2\left(x_{1},-k_{0} x_{1}-y_{0}\right)=\left(0, y_{0}\right)$. In other words, $\left(x_{1}, y_{1}\right)$ is a $K$ rational 24-torsion point of $E$.

The computation process explained as above thus gives our result immediately.

4.3. The case $E(K)_{\text {tors }}=\mathbb{Z} / 5 \mathbb{Z} \oplus \mathbb{Z} / 5 \mathbb{Z}$.

Theorem 4.4. Let $K=\mathbb{Q}\left(\zeta_{5}\right)$ with $\zeta_{5}$ a primitive fifth root of unity, and let $E$ be an elliptic curve over $K$ defined by

$$
E: y^{2}=x^{3}-a x+b
$$

where

$$
\left\{\begin{array}{l}
a=\frac{t^{20}-228 t^{15}+494 t^{10}+228 t^{5}+1}{48}, \\
b=\frac{t^{30}+522 t^{25}-10005 t^{20}-10005 t^{10}-522 t^{5}+1}{864},
\end{array}\right.
$$

with nonzero $t$ in $\mathbb{Q}$. Then $E(K)_{\text {tors }}$ is equal to $\mathbb{Z} / 5 \mathbb{Z} \oplus \mathbb{Z} / 5 \mathbb{Z}$ for almost all $t$.

Proof. The result follows from [15, Section 1.2]. 
4.4. The case $E(K)_{\text {tors }}=\mathbb{Z} / 2 \mathbb{Z} \oplus \mathbb{Z} / 14 \mathbb{Z}$.

Theorem 4.5. Let $b_{14}, c_{14}$ and $d_{14}$ be given in Table 3 , and let $E$ be an elliptic curve defined by the following equation:

$$
y^{2}+\left(1-c_{14}\right) x y-b_{14} y=x^{3}-b_{14} x^{2} .
$$

Then the torsion subgroup of $E$ over a quartic number field $K$ is equal to $\mathbb{Z} / 2 \mathbb{Z} \oplus$ $\mathbb{Z} / 14 \mathbb{Z}$ for almost all $t$ in $\mathbb{Q}$, where $K=\mathbb{Q}\left(\sqrt{A+B \sqrt{d_{14}}}\right)$ with

$$
\left\{\begin{array}{l}
A=A(t)=2\left(t^{2}-1\right)^{2}\left(t^{8}+8 t^{7}+24 t^{6}+32 t^{5}+4 t^{4}-32 t^{3}-24 t^{2}+8 t+2\right), \\
B=B(t)=-2 t\left(t^{2}-1\right)\left(t^{7}+7 t^{6}+16 t^{5}+10 t^{4}-18 t^{3}-26 t^{2}+12\right) .
\end{array}\right.
$$

Proof. Let $k=\mathbb{Q}\left(\sqrt{d_{14}}\right)$. Then the elliptic curve $E$ defined by the form in Eq. (15) has a $k$-rational point $P=(0,0)$ of order 14 by Theorem 3.1. Writing Eq. (15) as $y^{2}=f(x)$ with a cubic polynomial $f(x)$, we have that $f(x)$ has a linear factor over $k$ because $E$ has a $k$-rational point $7 P$ of order 2. Using similar reasoning as in the proof of Theorem 3.2 $E$ has full 2-torsion over an extension field $L$ of $k$ if and only if $A+B \sqrt{d_{14}}$ is a square in $L$. Hence the torsion subgroup of $E$ is $\mathbb{Z} / 2 \mathbb{Z} \times \mathbb{Z} / 14 \mathbb{Z}$ over the quartic number field $K$.

\subsection{The case $E(K)_{\text {tors }}=\mathbb{Z} / 2 \mathbb{Z} \oplus \mathbb{Z} / 16 \mathbb{Z}$.}

Theorem 4.6. Let $b_{16}, c_{16}$ and $d_{16}$ be given in Table 3, and let $E$ be an elliptic curve defined by the following equation:

$$
y^{2}+\left(1-c_{16}\right) x y-b_{16} y=x^{3}-b_{16} x^{2} .
$$

Then the torsion subgroup of $E$ over a quartic number field $K$ is equal to $\mathbb{Z} / 2 \mathbb{Z} \oplus$ $\mathbb{Z} / 16 \mathbb{Z}$ for almost all $t$ in $\mathbb{Q}$, where $K=\mathbb{Q}\left(\sqrt{A+B \sqrt{d_{16}}}\right)$ with

$$
\left\{\begin{aligned}
A= & A(t)=2\left(t^{2}+2 t-1\right)\left(t^{2}-2 t-1\right)\left(t^{16}+8 t^{15}+24 t^{14}+32 t^{13}+12 t^{12}-24 t^{11}-52 t^{10}-48 t^{9}-10 t^{8}\right. \\
& \left.+24 t^{7}+32 t^{6}+16 t^{5}-2 t^{4}-8 t^{3}-4 t^{2}+1\right), \\
A= & B(t)=-2(t+1)\left(t^{2}+2 t-1\right)\left(t^{2}-2 t-1\right)\left(t^{4}+2 t^{3}-1\right)\left(t^{8}+4 t^{7}+4 t^{6}-2 t^{4}-4 t^{3}-2 t^{2}+1\right) .
\end{aligned}\right.
$$

Proof. The proof is the same as in Theorem 4.5.

4.6. The case $E(K)_{\text {tors }}=\mathbb{Z} / 2 \mathbb{Z} \oplus \mathbb{Z} / 18 \mathbb{Z}$.

Theorem 4.7. Let $b_{18}, c_{18}$ and $d_{18}$ be given in Table 3 , and let $E$ be an elliptic curve defined by the following equation:

$$
y^{2}+\left(1-c_{18}\right) x y-b_{18} y=x^{3}-b_{18} x^{2} .
$$

Then the torsion subgroup of $E$ over a quartic number field $K$ is equal to $\mathbb{Z} / 2 \mathbb{Z} \oplus$ $\mathbb{Z} / 18 \mathbb{Z}$ for almost all $t$ in $\mathbb{Q}$, where $K=\mathbb{Q}\left(\sqrt{A+B \sqrt{d_{18}}}\right), A=A(t)$ is given by $A(t)=2(t-1)\left(t^{2}-t+1\right)\left(t^{81}-60 t^{80}+1823 t^{79}-37283 t^{78}+575948 t^{77}-7153345 t^{76}\right.$

$+74270830 t^{75}-661972936 t^{74}+5162951498 t^{73}-35748416786 t^{72}+222220017978 t^{71}$

$-1251268732638 t^{70}+6428384983229 t^{69}-30312842608758 t^{68}+131851626338239 t^{67}$

$-531250231119487 t^{66}+1989823103024944 t^{65}-6949508334581021 t^{64}+22691031914247536 t^{63}$

$-69421587124788592 t^{62}+199398166568254427 t^{61}-538599074700096200 t^{60}+1370123432130051153 t^{59}$

$-3286607755162234569 t^{58}+7442130833595477585 t^{57}-15922314702385616367 t^{56}$

$+32211496441342160295 t^{55}-61658320854407144771 t^{54}+111732077832460006205 t^{53}$

$-191757358447486495265 t^{52}+311783981857223273273 t^{51}-480377955812187198067 t^{50}$

$+701460096702393266510 t^{49}-970828388582220808403 t^{48}+1273499554107320498366 t^{47}$

$-1583214470476057699882 t^{46}+1865115992455672404654 t^{45}-2081672644416495160786 t^{44}$

$+2200653935514819200850 t^{43}-2202900614029732113706 t^{42}+2087342688173047087363 t^{41}$ 


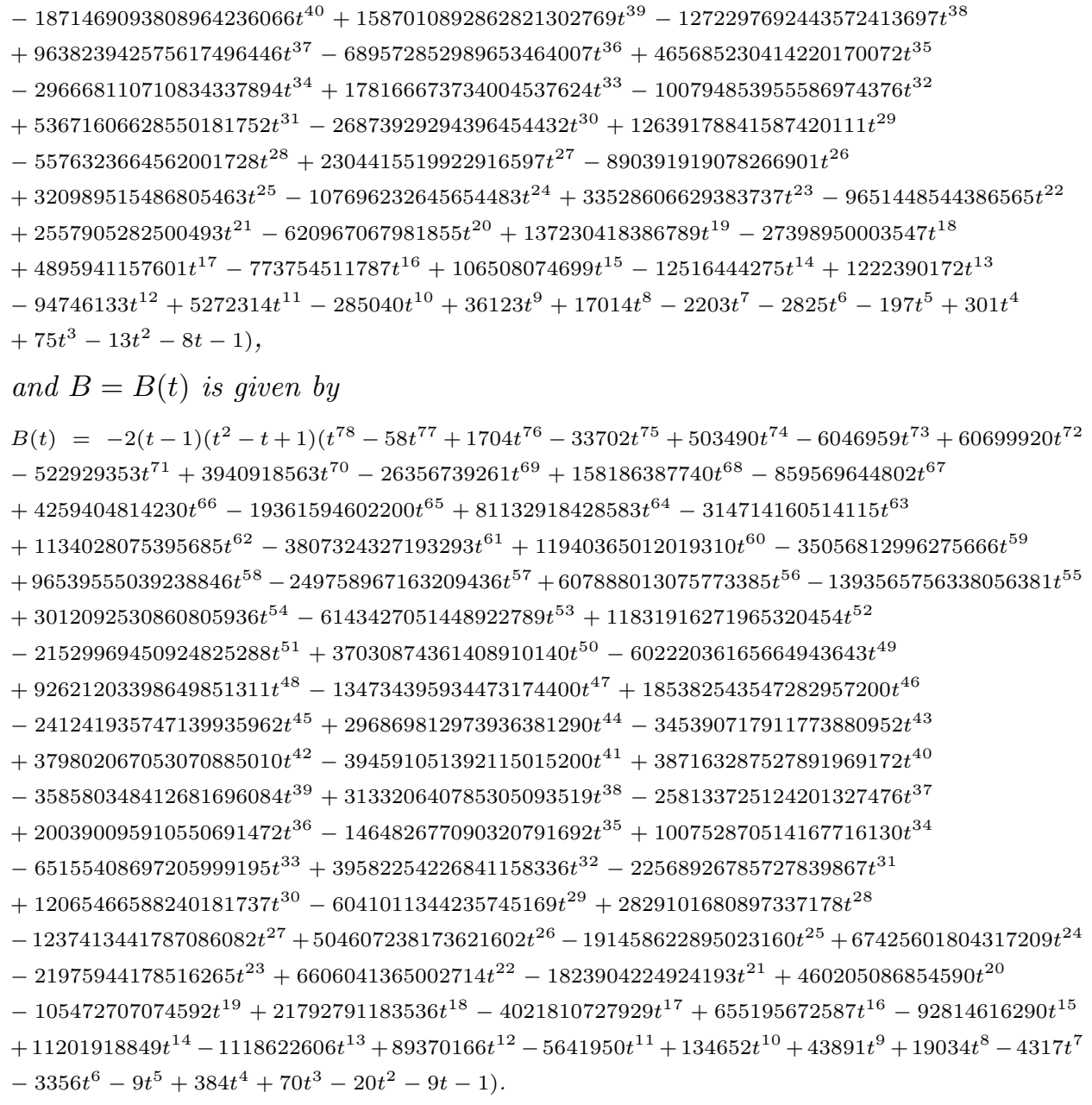

Proof. The proof is the same as in Theorem 4.5

4.7. The case $E(K)_{\text {tors }}=\mathbb{Z} / 3 \mathbb{Z} \oplus \mathbb{Z} / 9 \mathbb{Z}$. We need the following result in [10, Proposition 3.1] for proving Theorem 4.9, which is useful for finding the divisibility condition of a given point on $E$ by 3 .

Proposition 4.8. Let $K$ be a number field whose characteristic is different from 3 and which contains a primitive third root of unity $\zeta_{3}$. Let $E$ be an elliptic curve over $K$ with full 3-torsion given by $E: X^{3}+Y^{3}+Z^{3}=3 \mu X Y Z$, and $f_{S}=$ $-27\left(\mu^{3}-1\right) \frac{\zeta_{3}^{2} X+\zeta_{3} Y+\mu Z}{X+Y+\mu Z}$ and $f_{T}=9\left(\mu^{2}+\mu+1\right) \frac{X+\mu Y+Z}{X+Y+\mu Z}$ with $\mu^{3} \neq 1$. Then for the injection

$$
E(K) / 3 E(K) \rightarrow H^{1}(G, E[3]) \simeq K^{*} / K^{* 3} \times K^{*} / K^{* 3},
$$

where $H^{1}(G, E[3])$ is the Galois cohomology group, $G=\operatorname{Gal}(\bar{K} / K)$ and $E[3]=$ $E[3](\bar{K})$, the pair $\left(f_{S}, f_{T}\right)$ of rational functions on $E$ gives its image in $H^{1}(G, E[3])$ $\simeq K^{*} / K^{* 3} \times K^{*} / K^{* 3}$ when evaluated at a point of $E(K)$. 
Theorem 4.9. Let $K=\mathbb{Q}\left(\sqrt{3 t\left(4-t^{3}\right)}, \sqrt{-3}\right)$ with $t \in \mathbb{Q}$, and let $E$ be an elliptic curve defined by the equation

$$
X^{3}+Y^{3}+Z^{3}=3 \mu X Y Z,
$$

where $\mu=\zeta_{3}+\frac{\left(3 t^{2} \pm \sqrt{3 t\left(4-t^{3}\right)}\right)^{3}}{72 \sqrt{-3} t^{3}}$ with $\mu^{3} \neq 1$. Then the torsion subgroup of $E$ over a quartic number field $K$ is equal to $\mathbb{Z} / 3 \mathbb{Z} \oplus \mathbb{Z} / 9 \mathbb{Z}$ for almost all $t$.

Proof. We use Proposition 4.8. We note that $P=(0 ;-1 ; 1)$ is a 3 -torsion point from [1, Ch. 4, Section 2] so that we have $f_{S}=-27\left(\mu^{2}+\mu+1\right)\left(\mu-\zeta_{3}\right)$ and $f_{T}=$ $-9\left(\mu^{2}+\mu+1\right)$ when $f_{S}, f_{T}$ are evaluated at the point $P$. From Proposition 4.8 , it suffices to find a condition of $\mu$ for which $f_{S}$ and $f_{T}$ are cubic in some quartic number fields. Note that $\frac{f_{S}}{f_{T}}=3\left(\mu-\zeta_{3}\right)$. Setting $-3\left(\mu-\zeta_{3}\right)=x^{3}$ and $-3\left(\mu-\zeta_{3}^{2}\right)=y^{3}$ yields $f_{T}=-9\left(\mu-\zeta_{3}\right)\left(\mu-\zeta_{3}^{2}\right)=(-x)^{3} y^{3}$. We also have $x^{3}-y^{3}=3\left(\zeta_{3}-\zeta_{3}^{2}\right)=3 \sqrt{-3}$, so

$$
\left(-\frac{x}{\sqrt{-3}}\right)^{3}-\left(-\frac{y}{\sqrt{-3}}\right)^{3}=1
$$

Let $X=-\frac{x}{\sqrt{-3}}$ and $Y=-\frac{y}{\sqrt{-3}}$, then we have $X^{3}-Y^{3}=1$, and it is enough to show that the equation $X^{3}-Y^{3}=1$ has infinitely many quadratic points. Let $X=Y+t$, then we have

$$
3 t Y^{2}+3 t^{2} Y+t^{3}=1
$$

Then

$$
Y=\frac{-3 t^{2} \pm \sqrt{3 t\left(4-t^{3}\right)}}{6 t}
$$

Thus $X=Y+t$ and $Y$ are defined over quartic number fields $K$, so the result follows.

4.8. The case $E(K)_{\text {tors }}=\mathbb{Z} / 4 \mathbb{Z} \oplus \mathbb{Z} / 8 \mathbb{Z}$.

Theorem 4.10. Let $K=\mathbb{Q}\left(\sqrt{-1}, \sqrt{t^{4}-6 t^{2}+1}\right)$ with $t \in \mathbb{Q}$ and $t \neq 0, \pm 1$, and let $E$ be an elliptic curve defined by the equation

$$
y^{2}+x y-\left(\nu^{2}-\frac{1}{16}\right) y=x^{3}-\left(\nu^{2}-\frac{1}{16}\right) x^{2},
$$

where $\nu=\frac{t^{4}-6 t^{2}+1}{4\left(t^{2}+1\right)^{2}}$ and $t \neq 0, \pm 1$. Then the torsion subgroup of $E$ over $K$ is equal to $\mathbb{Z} / 4 \mathbb{Z} \oplus \mathbb{Z} / 8 \mathbb{Z}$ for almost all $t$.

Proof. Let $E$ be defined as in Subsection 3.6. Then the torsion subgroup of $E$ over $\mathbb{Q}(i)$ is equal to $\mathbb{Z} / 4 \mathbb{Z} \oplus \mathbb{Z} / 4 \mathbb{Z}$ whenever $\nu$ is a square. Note that $P=(0,0)$ is a point of order 4 and $2 P=\left(\nu^{2}-\frac{1}{16}, 0\right)$. There are two other 2 -torsion points $\left(-\frac{1}{8}+\frac{\nu}{2}, \frac{(4 \nu-1)^{2}}{32}\right)$ and $\left(-\frac{1}{8}-\frac{\nu}{2}, \frac{(4 \nu-1)^{2}}{32}\right)$. Let $\alpha=\nu^{2}-\frac{1}{16}, \beta=-\frac{1}{8}+\frac{\nu}{2}$ and $\gamma=-\frac{1}{8}-\frac{\nu}{2}$. By Theorem 2.1, there exist a $K$-rational point $Q$ with $2 Q=P$ if and only if $0-\alpha=-\left(\nu^{2}-\frac{1}{16}\right), 0-\beta=-\left(-\frac{1}{8}+\frac{\nu}{2}\right)$, and $0-\gamma=-\left(-\frac{1}{8}-\frac{\nu}{2}\right)$ are all squares in $K$. This follows from taking $\nu=\frac{t^{4}+6 t^{2}+1}{4\left(t^{2}+1\right)^{2}}$. 
4.9. The case $E(K)_{\text {tors }}=\mathbb{Z} / 6 \mathbb{Z} \oplus \mathbb{Z} / 6 \mathbb{Z}$.

Theorem 4.11. Let $K=\mathbb{Q}\left(\sqrt{-3}, \sqrt{8 t^{3}+1}\right)$ with $t \in \mathbb{Q}$, and let $E$ be an elliptic curve defined by the equation

$$
E_{\mu}: y^{2}=x^{3}-27 \mu\left(\mu^{3}+8\right) x+54\left(\mu^{6}-20 \mu^{3}-8\right)
$$

where $\mu=\frac{2 t^{3}+1}{3 t^{2}}$ with $t \neq 0,1,-\frac{1}{2}$. Then the torsion subgroup of $E$ over $K$ is equal to $\mathbb{Z} / 6 \mathbb{Z} \oplus \mathbb{Z} / 6 \mathbb{Z}$ for almost all $t$.

Proof. The curve given in Subsection 3.5 has the following Weierstrass model [15]

$$
E_{\mu}: y^{2}=x^{3}-27 \mu\left(\mu^{3}+8\right) x+54\left(\mu^{6}-20 \mu^{3}-8\right) .
$$

If $\mu=\frac{2 t^{3}+1}{3 t^{2}}$, then $E_{\mu}$ has $\mathbb{Z} / 6 \mathbb{Z} \oplus \mathbb{Z} / 6 \mathbb{Z}$ as its full torsion group over $K=$ $\mathbb{Q}\left(\sqrt{-3}, \sqrt{8 t^{3}+1}\right)$. We note that $E_{\mu}$ has the following 2-torsion points: $\left(\left(8 t^{6}+\right.\right.$ $\left.\left.20 t^{3}-1 \pm 3 \sqrt{\left(8 t^{3}+1\right)^{3}}\right) / 6 t^{4}, 0\right), \quad\left(-\left(8 t^{6}+20 t^{3}-1\right) / 3 t^{4}, 0\right)$.

\section{ACKNOWLEDGMENTs}

The authors would like to thank KIAS (Korea Institute for Advanced Study) for its hospitality. We are also grateful to Filip Najman for pointing out an error in the computation of subsection 4.8 and fixing it. Finally, we thank anonymous referees for their helpful comments, which improved the clarity of this paper.

\section{REFERENCES}

1. D. Husemöller, Elliptic curves, Second edition, Springer-Verlag, New York, 2004. MR2024529 (2005a:11078)

2. D. Jeon, C.H. Kim and Y. Lee, Families of elliptic curves over cubic number fields with prescribed torsion subgroups, Math. Comp. 80 (2011), 579-591. MR.2728995

3. D. Jeon, C.H. Kim and E. Park, On the torsion of elliptic curves over quartic number fields, J. London Math. Soc. (2) 74 (2006), 1-12. MR2254548 (2007m:11079)

4. D. Jeon, C.H. Kim and A. Schweizer, On the torsion of elliptic curves over cubic number fields. Acta Arith. 113 (2004), 291-301. MR2069117 (2005f:11112)

5. S. Kamienny and B. Mazur, Rational torsion of prime order in elliptic curves over number fields. With an appendix by A. Granville. Columbia University Number Theory Seminar (New York, 1992). Astérisque No. 228 1995, 3, 81-100. MR1330929 (96c:11058)

6. A.W. Knapp, Elliptic Curves, Mathematical Notes 40, Princeton University Press, Princeton, NJ, 1992. MR1193029 (93j:11032)

7. D.S. Kubert, Universal bounds on the torsion of elliptic curves, Proc. London Math. Soc. (3) 33 (1976), 193-237. MR0434947(55:7910)

8. B. Mazur, Modular curves and the Eisenstein ideal, Publ. Math. I.H.E.S. 47 (1977), 33-168. MR 488287 (80c:14015)

9. L. Merel, Bornes pour la torsion des courbes elliptiques sur les corps de nombres, Invent. Math. 124 (1996), no 1-3, 437-449. MR.1369424(96i:11057)

10. C. O'Neil, Explicit descent over $X(3)$ and $X(5)$, arXiv:0201.5321 [math.NT] (2002).

11. A. Petho, T. Weis and H.G. Zimmer, Torsion groups of elliptic curves with integral $j$-invariant over general cubic number fields, Internat. J. Algebra Comput. 7 (1997), 353-413. MR 1448331 (98e:11069)

12. P. Parent, No 17-torsion on elliptic curves over cubic number fields, J. Theor. Nombres Bordeaux 15 (2003), 831-838. MR2142238 (2006a:11071)

13. P. Parent, Torsion des courbes elliptiques sur les corps cubiques, Ann. Inst. Fourier (Grenoble) 50 (2000), no. 3, 723-749. MR1779891 (2001i:11067)

14. M.A. Reichert, Explicit determination of nontrivial torsion structures of elliptic curves over quadratic number fields, Math. Comp. 46 (1986), 637-658. MR829635 (87f:11039) 
15. K. Rubin and A. Silverberg, Families of elliptic curves with constant mod $p$ representations, in Elliptic curves, modular forms, and Fermat's last theorem (Hong Kong, 1993), 148-161, Ser. Number Theory, I, Int. Press, Cambridge, MA, 1995. MR.1363500 (96j:11078)

16. J. Silverman, The arithmetic of elliptic curves, Springer-Verlag, New York, 1986. MR817210 (87g:11070)

17. A.V. Sutherland, Constructing elliptic curves over finite fields with prescribed torsion, arXiv:0811.0296v2 [math.NT] (2008).

18. H.G. Zimmer, Torsion groups of elliptic curves over cubic and certain biquadratic number fields, Arithmetic geometry (Tempe, AZ, 1993), 203-220, Comtemp. Math., 174, Amer. Math. Soc., Providence, RI, 1994. MR1299744 (95i:11056)

Department of Mathematics Education, Konguu National University, Kongu, ChungNAm, SOUTh Korea

E-mail address: dyjeon@kongju.ac.kr

Department of mathematics and Research institute for NATURAl SCiences, Hanyang University, Seoul, South Korea

E-mail address: chhkim@hanyang.ac.kr

Department of Mathematics, Ewha Womans University, Seoul, South Korea

E-mail address: yoonjinl@ewha.ac.kr 